\title{
What ICA Provides for ECG Processing: Application to Noninvasive Fetal ECG Extraction
}

\author{
Reza Sameni ${ }^{1,2}$, Christian Jutten ${ }^{1}$, Mohammad B. Shamsollahi ${ }^{2}$ \\ 1- Laboratoire des Images et des Signaux (LIS) - CNRS UMR 5083, INPG, UJF, Grenoble, France \\ 2- Biomedical Signal and Image Processing Laboratory (BiSIPL), School of Electrical Engineering, \\ Sharif University of Technology, Tehran, Iran \\ Emails: reza.sameni@lis.inpg.fr, christian.jutten@lis.inpg.fr, mbshams@sharif.ir
}

\begin{abstract}
In recent studies, Independent Component Analysis (ICA) has been used for the analysis of multi-channel ECG recordings. However most of these works have been carried out from the signal processing perspective. In this work, the single dipole vector theory of the heart and the ECG dimensionality are studied from the source separation viewpoint. Based on this study, the interpretation of the components extracted from multi-channel ECG and maternal abdominal recordings, and their relationship with the vectorcardiogram representation of the cardiac dipole are presented. The results of this study can be used for the extraction of meaningful clinical indexes, based on ICA techniques.
\end{abstract}

\section{INTRODUCTION}

Statistical decomposition techniques such as the Principal Component Analysis (PCA) [1], Singular Value Decomposition (SVD) [2], and Independent Component Analysis (ICA) [3], [4], [5] have been widely used as promising methods of multichannel ECG analysis, and noninvasive fetal ECG extraction. However, the research in this area has been mainly carried out from the signal processing point of view, and there are few works on the physiological interpretations of the extracted components. Moreover, there are many issues such as the stability, robustness, and noise-sensitivity of the extracted components which are left as open problems.

In this paper, it is shown that by a re-study of the origins of the ECG signals and their relationship with other representations of the cardiac activity such as the Vectorcardiogram (VCG), it is possible to relate the shapes of the extracted components with their physiological counterpart. This study is hoped to shed light onto the application of source separation techniques for the extraction of better cardiac indexes.

The rest of the paper is organized as follows. In section II the physiological bases of the single dipole model of the heart, and its relationship with the potentials recorded from the body surface are reviewed. In section III the dimensionality of the ECG signals are discussed in more details. Based on these backgrounds, the interpretation of the components extracted from multi-channel ECG, maternal abdominal recordings, and some useful clinical indexes will be presented in sections IV to VI. In the last two sections some numerical results and concluding remarks are presented.

\section{The Vectorcardiogram Vs. THE ELECTROCARDIOGRAM}

The electrical nervous system of the heart which enables the contraction of the cardiac muscle is distributed throughout the heart. The resultant electrical activity of the heart has been modeled with various methods ranging from single dipole models to activation maps [6]. Among these methods, the simplest and yet the most popular is the single dipole model which is believed to explain $80 \%-90 \%$ of the representation power of the body surface potentials [7], and the ECG and VCG are based on it. According to the single dipole model, the cardiac electrical activity may be represented by a timevarying rotating vector, the origin of which is assumed to be at the center of the heart and its end sweeps a quasi-periodic region in the space. This vector may be mathematically represented in the Cartesian coordinates, as follows:

$$
\mathbf{d}(t)=x(t) \hat{\mathbf{i}}+y(t) \hat{\mathbf{j}}+z(t) \hat{\mathbf{k}},
$$

where $\hat{\mathbf{i}}, \hat{\mathbf{j}}$, and $\hat{\mathbf{k}}$ are the unit vectors of the Cartesian coordinates.

The dipole model is a means of modeling the heart source, and in order to model the electrical recordings on the body surface, an additional model is required for the propagation of the heart potentials in the thorax volume conductor. By assuming this volume conductor as a passive resistive media which only attenuates the source field, any ECG signal would simply be a linear projection of $\mathbf{d}(t)$, onto the direction of the recording electrode axes $\mathbf{v}=a \hat{\mathbf{i}}+b \hat{\mathbf{j}}+c \hat{\mathbf{k}}$ :

$$
E C G(t)=<\mathbf{d}(t), \mathbf{v}>=a \cdot x(t)+b \cdot y(t)+c \cdot z(t),
$$

A 3-D vector representation of the ECG, namely the Vectorcardiogram (VCG), is also possible by using three of such ECG signals. Basically any three linearly independent set of ECG electrodes can be used to construct the VCG, but in order to achieve an orthonormal representation which best resembles the dipole vector $\mathbf{d}(t)$, a set of three orthogonal electrodes which best correspond to the three body axes are selected. The normality of the representation is further achieved by attenuating the different leads with a priori knowledge of the body volume conductor, to compensate for the nonhomogeneity of the body thorax. The Frank lead system, or the 
corrected Frank lead system which have better orthogonality and normalization, are conventional means of recording the VCG [8].

The quasi-periodic VCG shape basically consists of three loops which correspond to the $\mathrm{P}-$, QRS-, and the T-waves of the ECG. These loops intersect with each other in the isoelectric point of heart where the heart is electrically at rest. The QRS-loop is the most dominant loop and the P-loop is more or less in the same plane but with a much smaller radius. The angles, sizes, and curvature of each of these loops are known to convey physiological information which is not easily seen in the multi-channel ECG representation.

The ECG recorded from the body surface is contaminated with different noises such as muscle artifacts, electrode/body movements, and baseline wanders [9]. The baseline wander is mainly due to the impedance changes of the body volume conductor during respiration, and it causes a translation of the isoelectric point of the cardiac signals in multi-dimensional VCG space, together with rotation and scaling of the VCG loop.

\section{Dimensionality of THE ECG}

The study of dimensionality of multivariate data is a wellknown and yet challenging issue in statistical data analysis, and there are several statistical and geometrical approaches developed for the dimension estimation and reduction for multivariate data. Perhaps the most popular statistical tool for dimension estimation and reduction is the Principal Component Analysis (PCA) and some of its nonlinear variants [10].

The main limitation of dimension estimation is that all practical data recordings contain measurement noise, and the higher order dimensions are very likely to correspond to the noise components, rather than the signal. Thus the discrimination of the signal and noise dimensions is not straightforward. For ECG signals, based on the single dipole model of the heart, Dower $e t a l$ have developed a transformation for finding the standard 12-lead ECGs from the Frank electrodes [11]. The Dower transform is simply a $12 \times 3$ linear transformation between the standard 12-lead ECGs and the Frank leads, which can be found from the Minimum Mean Square Error (MMSE) estimate of a transformation matrix between the two electrode sets. Apparently the transformation is influenced by the standard locations of the recording leads and the attenuations of the body volume conductor, respecting to each electrode [12].

The Dower transform and its inverse [13], are evident results of the single dipole model of the heart with a linear propagation model of the body volume conductor, suggesting that the heart has only three geometrically independent dimensions, and any ECG signal recorded from the body surface would be a linear combination of the same three sources. However since the single dipole model of the heart is not a perfect representation of the cardiac activity, cardiologists usually use more than three ECG electrodes (between 6 to 12) to study the cardiac activity [8]. The problem of ECG dimensionality is generally a subjective issue, but our later presented results show that between 4 to 6 statistically uncorrelated dimensions are required to represent typical ECG signals. This result is with accordance with the conventional measurements made by cardiologists, but in contrast with some previous works on statistical ECG decomposition which only consider 3 dimensions [3].

\section{INTERPRETATION OF INDEPENDENT COMPONENTS EXTRACTED FROM THE ECG}

The simplest form of the Independent Component Analysis (ICA) problem consists of the estimation of independent sources $s_{i}(t)(i=1, \ldots N)$, from their blind linear mixtures:

$$
\mathbf{x}(t)=A \cdot \mathbf{s}(t)
$$

where $\mathbf{x}(t)=\left[x_{1}(t), \ldots, x_{n}(t)\right]^{T}, \mathbf{s}(t)=\left[s_{1}(t), \ldots, s_{n}(t)\right]^{T}$, and $A$ is the mixing matrix. Reviewing the general steps of typical ICA algorithms, the mean of the data vector $\mathbf{x}(t)$ is removed, the data is decorrelated and whitened. The whitened data is further rotated by an orthogonal transformation, in order to achieve the independent components (ICs) [14].

Mathematically the ICA problem of (3) almost always gives a solution for the most independent sources. However when applying the ICA to multivariate ECG recordings, the interpretation of the extracted sources and the extraction of meaningful clinical measures from these components need a closer study of the ECG and the VCG.

Following the explanations of previous sections, it should be first of all noted that the formulation in (3) should not be mistaken with (2). In fact, unlike the independent decomposition of (3), the three geometrically orthogonal components of the ECG are not statistically independent. This suggests that the ICs extracted from multi-channel ECGs are not necessarily limited to three components and may correspond to other meaningful components.

Regarding the explanations of section II, it is evident that the multivariate ECG recordings are the samples of a quasiperiodic loop in the multi-dimensional space, and by applying ICA we are trying to find the directions conveying the most information in this space. Intuitively these directions can be thought to have some correspondence with the VCG-loops and planes, since the multi-dimensional space spanned by the heart dipole vector is rather sparse and follows a rather quasiperiodic loop in the space.

Before giving an interpretation for the extracted ICs, the isoelectric point of the heart needs to be further discussed. The ECG and VCG signals are not symmetric around their mean values, and the isoelectric point of the heart slightly differs from the mean values of the data. Meanwhile, an implicit assumption of common ICA algorithms is that the input signals are symmetric around their mean values since the mean of the data is initially removed, and the sign of the ICs are not determinable [14]. This means that when applying conventional ICA algorithms to signals such as the ECG (without considering the data asymmetry), the extracted ICs do not correspond to the intuitively expected directions in the VCG space. 
As an illustration, the distribution plot of an asymmetric dataset is depicted in Fig. 1-a. The columns of the mixing matrix, extracted by the JADE ICA algorithm ([14]) have also been depicted. As seen in this figure, due to the data asymmetry, ICA does not give the directions of the two lobes of the distribution. Similarly, for the problem of ECG signals we are more interested in the directions in which the data has the most variations from the isoelectric point, rather than the true ICs.

A rather simple, but effective, method to solve this problem is to remove the baseline wander of the ECG (which is identical to making the isoelectric point stationary in the multidimensional VCG space), and then virtually make the data zero-mean with respect to the isoelectric point, by augmenting the data samples with their minus values:

$$
\mathbf{x}_{n \times 2 T} \leftarrow\left[\mathbf{x}_{n \times T},-\mathbf{x}_{n \times T}\right]
$$

By this way, we are adding the image of the current dataset to the distribution space, and making the density function of the samples symmetric. This causes the dataset and the processing time twice as long, but has the benefit that the ICs are found with respect to the isoelectric point of the $\mathrm{VCG}^{1}$. The result of applying this idea to the data of Fig. 1-a, and the new columns of the mixing matrix extracted by the JADE algorithm are shown in Fig. 1-b. This simple idea may also be useful in other applications, to exploit the local behaviors of complex multivariate distributions around any point other than the true data mean value.

We can now state that for a typical VCG loop having small inter-beat variations, by making the data symmetric around the isoelectric point, it is reasonable to assume that the columns of the mixing matrix extracted by ICA correspond to the most representative axes of the VCG loop scatter. These orientations usually correspond to the directions of the main planes in the VCG space. In terms of the ECG they also correspond to the main waves of ECG signals, meaning that the shapes and time delays between the extracted ICs convey information about the movements of the cardiac dipole vector as it rotates in space.

From the VCG perspective we can also explain why the baseline wanders of multi-channel ECG recordings, sometimes

${ }^{1}$ Evidently the second-half of the extracted sources will also be redundant in this case.

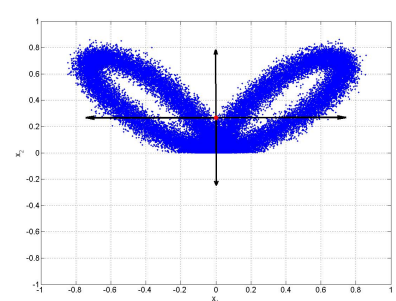

(a)

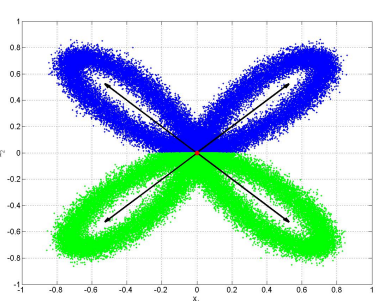

(b)
Fig. 1. The effect of PDF asymmetry on the extracted ICs. (a) asymmetric PDF (b) PDF made symmetric using equation (4). The mixing vectors extracted by ICA are plotted in each case. show up as independent components. In fact as the VCG loops are rather planar, the baseline wander components which drift the VCG loop in directions orthogonal to the QRSloop are more probable to be extracted as new independent components; but the baseline drifts which are in the same direction as the QRS-loop will not be detected as separate components and will be seen in the components of the QRSloop.

The intrinsic indeterminacy of scales and signs is a wellknown limitation of the ICA problem as defined in (3). By using ICA, we are estimating the heart sources and the mixing matrix corresponding to the body volume conductor at the same time. However due to the non-homogeneity of the body volume conductor the original heart signals are not attenuated with the same amount in all directions. This means that ICA cannot find the true scale of the heart sources without $a$ priori knowledge about the heart dipole vector. In conventional vectorcardiography, this problem is already known, and this was in fact the motivation behind the development of the corrected Frank lead systems [8]. To solve this ambiguity we can either consider a normalized canonical representation of the VCG loop, or otherwise assume an orthonormal mixing matrix.

\section{INDEPENDENT COMPONENTS EXTRACTED FROM ABDOMINAL RECORDINGS}

Multi-dimensional ICA, or Independent Subspace Analysis (ISA) is an extension of ICA used for the separation of independent subspaces, rather than independent components [4]. The privilege of ISA compared with ICA is that it can separate multiple groups of sources that can be dependent within each group but independent from the sources of other groups (like the subspace of the fetal ECG vs. the maternal ECG subspace). ISA has been approved as a promising tool for the extraction of fetal cardiac signals from maternal abdominal recordings. Yet most previous works in this area are based on the assumption that the maternal heart has only 3 statistically independent dimensions, while the fetal heart is statistically 2-dimensional. This assumption about the dimensionality of the fetal ECG may be practically true, due to the weakness of the fetal ECG components (about $1 / 30$ to $1 / 5$ of the maternal ECG amplitude), its distance from the abdominal electrodes, and the changes in the conductivity of the maternal volume conductor due to the development of the vernix caseosa layer around the fetus [1]. However, physiologically there is no reason for the dimensional difference between fetal and adult hearts, especially in the higher periods of the pregnancy.

In fact, with the discussions of section IV, the effect of the VCG isoelectric point becomes much more important for the combination of the fetal and maternal ECGs. As a simple illustration, an artificial mixture of the maternal and fetal VCG components is depicted in Fig. 2. Accordingly it is seen that the mean of the whole data distribution (indicated by a circle), is rather distanced from the fetal isoelectric point (indicated by a square). This means that by applying conventional ICA algorithms that find the ICs with respect to the mean of the 


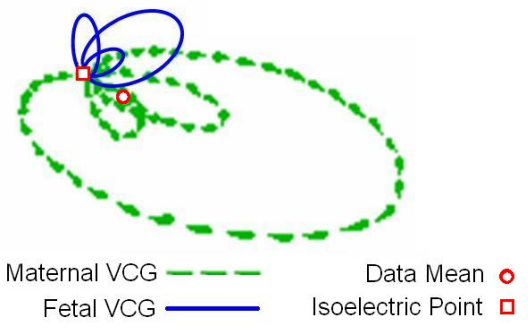

Fig. 2. Illustration of the maternal and fetal VCG mixtures

data, the details of the fetal VCG loop are not extracted as ICs, especially that the maternal components are much more dominant in these mixtures. So with the ideas of previous sections, we should be able to extract better details of the fetal heart components by finding the ICs with respect to the isoelectric point of VCG rather than the mean of the whole data. However, a question which arises at this point and requires further studies is whether the isoelectric points of the fetal and maternal VCGs can be assumed to be the same points in the distribution space of the recordings, and whether the baseline drifts of the fetal and maternal VCG loops are in the same directions.

\section{Vi. Extraction of Clinical Measures}

In previous works, different clinical indexes have been extract from the VCG loops [1], [15]. With the previous discussions on the interpretation of the ICs extracted from multi-channel ECG recordings, it is additionally possible to use the directions and variances of these ICs as measures of the surface of the main VCG loops and the volume of the VCG which are interesting for diagnostic applications. Moreover some other interesting clinical indexes such as the angle of the VCG loops and the angles between the T-loop and the QRS-loop are easily extractable from the ICs and their corresponding mixing matrix. The simultaneous recording of geometrically orthogonal leads such as the Frank leads also provide a reference for the study of the angle between the different loops, so that we can calculate the extracted angles with respect to the body planes.

\section{RESULTS}

\section{A. ECG dimensionality study}

Based on the previous discussions, the sensitivity of the different ECG components with respect to the data dimensions has been studied. For this, a simple dimension reduction based on PCA has been carried out on signals from the MIT-BIH PTB database [16]. This database contains 15 channels of ECG recordings, containing the standard 12-leads and the 3 Frank leads. Before applying the dimension reduction, the power of each channel was normalized, and the SNR of each of the P-, QRS-, and the T-segments of the ECG was calculated by comparing the original and the reduced dimension signals. As seen in Fig. 3, the P waves are the most sensitive portion of the ECG to dimension reduction, mainly due to their weakness.

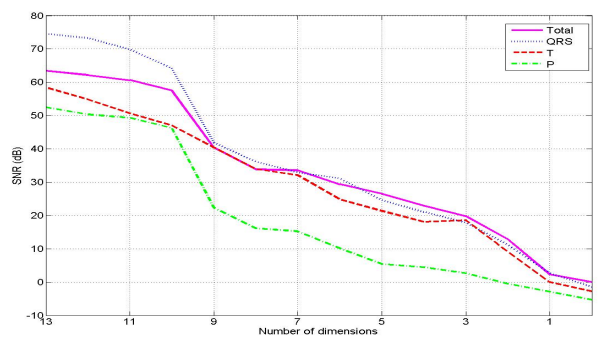

Fig. 3. Sensitivity of different ECG components to dimension reduction using PCA

According to these results, for an SNR value of $20 \mathrm{~dB}$, which is visually significant in the ECG plots, at least 5 dimensions are required; except for the $\mathrm{P}$-wave which requires 9 dimensions for the same SNR. Note that the sensitivity of the different channels to dimension reduction were not the same, and the results of Fig. 3 are an average of all the channels.

Another approach to estimate the data dimensions was the localized PCA. For this, the baseline wander of the data was removed and the isoelectric point of the dataset was found, and the data distribution was made symmetric according to (4). Then all the points which were within the distance of $r$ from the isoelectric point were selected and given to the PCA algorithm, and the number of dimensions required for the representation of different percentages of the whole signal energy were calculated from the eigenvalues of the data covariance matrix. The result of this study can be seen in Fig. 4 , where the required dimensions are plotted vs. the percentage of the total required energy $\left(P_{s}\right)$ for different values of the radius $r . P_{s}$ can also be translated to the $S N R$ achieved by keeping a certain number of dimensions for the samples within the distance of $r$. Considering the typical shape of the VCG, for small values of $r$ the eigenvalues are mainly due to noise, as they correspond to the fluctuations around the isoelectric point. While as $r$ increases, the true VCG components such as the $\mathrm{P}-, \mathrm{T}-$, and QRS-loops are considered in the eigenvalue calculations of the PCA. Considering the fact that the P-, and T-loops have smaller radius than the QRS-loop, it is evident that when $r$ is increased beyond the maximum radius of the P-loop ( $r \approx 0.5$ in Fig. 4), the further added information are only due to the T-, and the QRS-loops. The same statement is true for when $r$ exceeds the maximum of the T-loop radius, where any information beyond the T-loop limit $(r \approx 2.5$ in Fig. 4) will only correspond to the QRS-loop.

Another interesting result is achieved by studying the effect of a pre-processing dimension reduction stage using PCA on the robustness of the extracted ICs, in presence of Gaussian noise. For this study additive white Gaussian noise has been added to the signals after baseline wander removal and symmetrization of the data distribution. Next a PCA dimension reduction stage has been carried out, and the ICs have been extracted from the reduced dimension data. Knowing that the columns of the mixing matrix correspond to the axes of the mixing directions, the angles between these columns (or equivalently their inner product) calculated before and after 


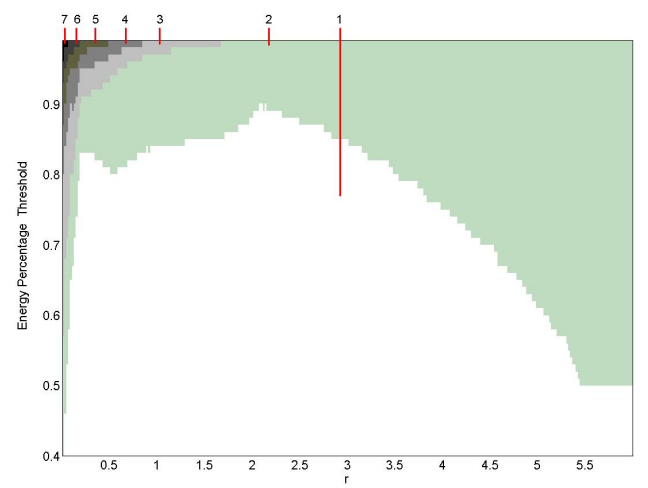

Fig. 4. Number of dimensions required for preserving a specific percentage of the signal energy, by applying a local PCA to the points within a radius of $r$ with respect to the isoelectric point of the VCG

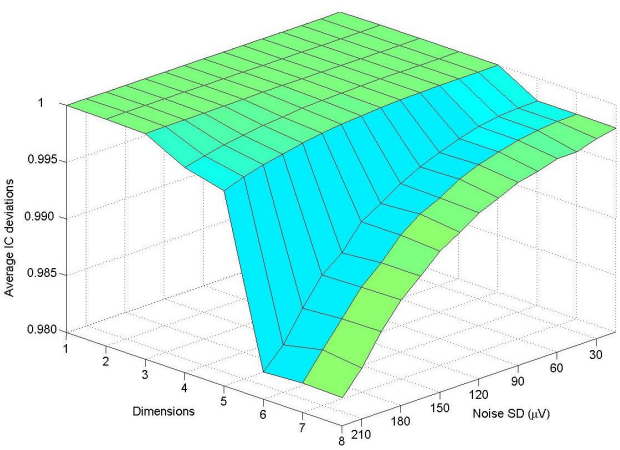

Fig. 5. Stability of the extracted independent components

adding noise, can be assumed as a measure of the robustness of each component (and the demixing matrix) with respect to noise. In Fig. 5, the average of the explained inner product is depicted vs. the noise standard deviation and the number of dimensions kept by $\mathrm{PCA}^{2}$. Accordingly, the first three dimensions are very robust to noise over the whole noise range of study, the $4^{\text {th }}$ and $5^{\text {th }}$ dimensions are sensitive to high values of noise, and as we increase the dimensions beyond 5 the extracted ICs become very sensitive to noise. Apparently the results approve that high dimensional ICs are more sensitive to noise and a well selected PCA dimension reduction stage tends to make the extracted ICs more robust to noise.

Following the discussions on the dimensionality of ECG signals, it is now evident that up to 5 robust ICs can be extracted from a typical ECG, which correspond to the main directions of the VCG loop, and convey physiologically meaningful information about the shape of these signals. In Fig. 6-a typical components which have been extracted from a typical ECG signal after performing the baseline wander removal can be seen. The VCG generated from the Frank lead electrodes, and the columns of the mixing matrix corresponding to the IC of Fig. 6-a are depicted in Fig. 6-b. As seen in this figure, the extracted components have time delays with respect to each other which correspond to the local peaks of the VCG-loop.

\footnotetext{
${ }^{2}$ Apparently, the indexes which are closer to 1 have been more robust to noise.
}

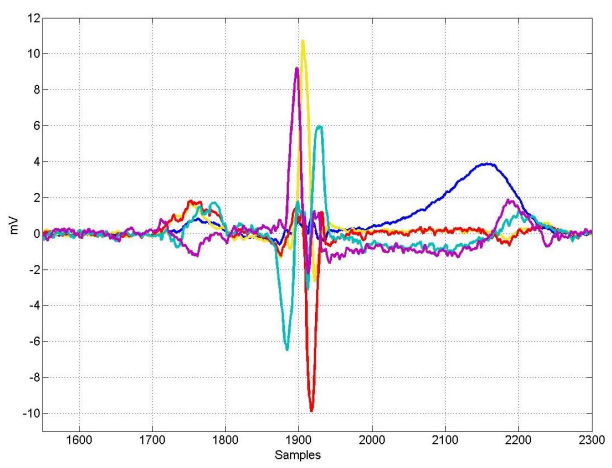

(a)

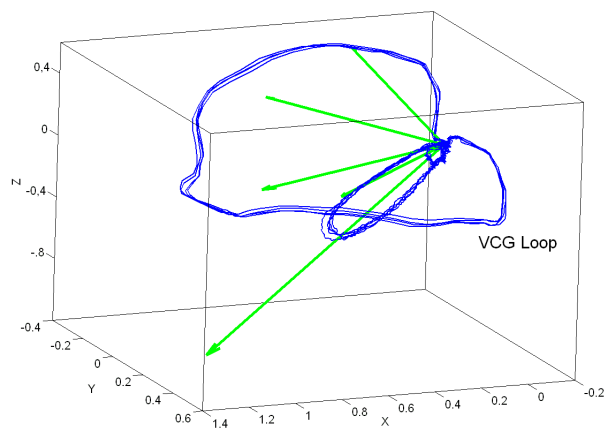

(b)

Fig. 6. (a) ICs extracted from multi-channel ECG recordings (Notice the time delay of the extracted component peaks), (b) The VCG representation of the Frank leads, together with the columns of the mixing matrix (or the mixing vectors)

\section{B. Fetal ECG extraction}

In order to extract fetal ECG components the DaISy database was used, which is a 10 second recording from 8 channels, containing 5 abdominal recordings and 3 thoracic recordings from a pregnant woman [17]. The data was preprocessed by removing the maternal baseline wander and by detecting the isoelectric point of the VCG. Then the JADE ICA algorithm was applied to the data. From the 8 extracted components, 4 clearly corresponded to the maternal heart, 2 with the fetal heart, and 2 with noise. However from the previous discussions it is clear that since the fetal components are much weaker than the maternal components, and since some of the fetal VCG loops can be co-planar with the maternal VCG planes, a complete extraction of the fetal and maternal subspaces (as assumed in [4], [3]) is not always achievable and some fetal ECG components might be found in the maternal components. To validate this assumption, the R-peaks of the fetal ECG were detected from the strongest fetal IC, and using these peaks a synchronous averaging was performed over all the ICs. The result of this averaging is plotted in Fig. 7. As it is seen in this figure, the synchronous averaging approves the existence of 5 to 6 distinct components in the fetal ECG (with significant time delays), which are not all extracted as individual components by ICA, as they lay within the maternal VCG subspaces. 


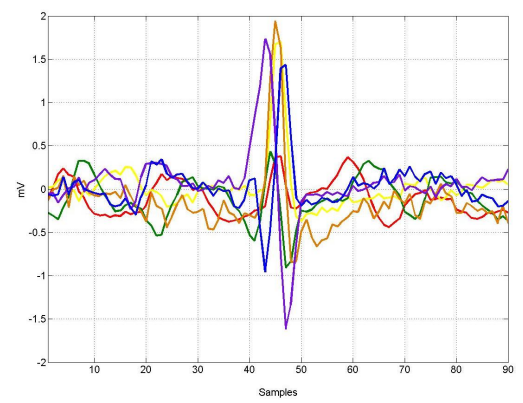

Fig. 7. Synchronous averaging of the extracted ICs, showing multiple components of the fetal ECG

\section{CONCLUSIONS}

In this paper, the application of ICA for the processing of ECG signals and issues concerning the dimensionality of these signals were studied. As it was discussed, the interpretation of the ICs extracted from multi-channel recordings is closely related to the VCG representation of these signals. Although the results of applying ICA to ECG recordings can be very subjective and database dependent, the following general results were hereby approved:

- The ICs extracted from multi-channel ECG signals very well correspond to the components of VCG loops. Moreover as the single dipole model of the heart and the assumption of a linear time-invariant body volume conductor model are not ideal description of the problem, the dimensions above 3 also convey physiologically meaningful information. As it was shown, by using ICA more than 3 (up to 5 or 6 ) robust components are extractable from the ECG which are time-synchronized with the rotation of the cardiac dipole.

- Baseline wanders of the ECG cause translations of the VCG loop. So the extraction of baseline wander as an IC highly depends on the direction of transition caused by the baseline wander, with respect to the VCG components.

- In order to have robust and stable components which convey information about the angles between VCG loops, it is necessary to find the components with respect to the isoelectric point of these loops.

- The exact detection of the isoelectric point of the VCG, VCG loop alignment, and baseline wander removal are even more important for the noninvasive extraction of fetal ECG components due to the weakness of the fetal components.

- As the fetal and maternal VCG loop components may become co-planar, even effective methods such as independent subspace decomposition are not guaranteed to extract all the fetal components. This also explains why there are usually no more than 1 or 2 ICs corresponding to the fetal heart. To overcome this problem, the sparsity of the ECG signal and the temporal information of the fetal and maternal ECGs are expected to be helpful.

Moreover recently developed filtering methods which use the temporal dynamics of the ECG signal can be used as a complement for the removal of the maternal components from the extracted ICs [18]. In fact, as it was discussed even the subspaces of the fetal and the maternal ECGs are not completely distinct. So by using dynamic filters, as proposed in [18], it should be possible to use a dynamic model that is time synchronous with the maternal ECG, to remove the maternal components while preserving the fetal components.

\section{ACKNOWLEDGMENT}

This work has been partially supported by the French Embassy in Iran (PAI Gundishapur), the Center for International Research and Collaboration (ISMO) in Iran, and the Iran Telecommunication Research Center (ITRC).

\section{REFERENCES}

[1] T. Oostendorp, Modeling the Fetal ECG. Ph.D. dissertation, K. U. Nijmegen, The Netherlands, 1989.

[2] P. P. Kanjilal, S. Palit, and G. Saha, "Fetal ECG extraction from singlechannel maternal ECG using singular value decomposition," IEEE Trans. Biomed. Eng., vol. 44, pp. 51-59, Jan. 1997.

[3] L. de Lathauwer, B. de Moor, and J. Vandewalle, "Fetal electrocardiogram extraction by blind source subspace separation," IEEE Trans. Biomed. Eng., vol. 47, pp. 567-572, May 2000.

[4] J.-F. Cardoso, "Multidimensional independent component analysis," in Proceedings of the IEEE International Conference on Acoustics, Speech, and Signal Processing (ICASSP '98), vol. 4, May 1998, pp. 1941-1944.

[5] F. Vrins, V. Vigneron, C. Jutten, and M. Verleysen, "Abdominal Electrodes Analysis by Statistical Processing for Fetal Electrocardiogram Extraction," in Proc. 2nd IASTED Int. Conf. on Biomedical Engineering (BioMed 2004), Innsbruck , Austria, February 16-18 2004, pp. 244-249.

[6] O. Dössel, "Inverse problem of electro- and magnetocardiography: Review and recent progress," Int. J. Bioelectromagnetism, vol. 2, no. 2, 2000. [Online]. Available: http://www.ijbem.org/volume2/number2/doessel/paper.htm

[7] A. van Oosterom, "Beyond the dipole; modeling the genesis of the electrocardiogram," 100 years Einthoven, pp. 7-15, 2002.

[8] J. A. Malmivuo and R. Plonsey, Eds., Bioelectromagnetism, Principles and Applications of Bioelectric and Biomagnetic Fields. Oxford University Press, 1995. [Online]. Available: http://butler.cc.tut.fi/ malmivuo/bem/bembook

[9] G. M. Friesen, T. C. Jannett, M. A. Jadallah, S. L. Yates, S. R. Quint, and H. T. Nagle, "A comparison of the noise sensitivity of nine QRS detection algorithms," IEEE Trans. Biomed. Eng., vol. 37, no. 1, pp. 85-98, 1990.

[10] T. Stamkopoulos, K. Diamantaras, N. Maglaveras, and M. Strintzis, "ECG analysis using nonlinear PCA neural networks for ischemia detection," IEEE Trans. Signal Processing, vol. 46, pp. 3058-3067, Nov. 1998.

[11] G. E. Dower, H. B. Machado, and J. A. Osborne, "On deriving the electrocardiogram from vectorcardiographic leads," Clin. Cardiol., vol. 3, p. 87, 1980.

[12] L. Hadzievski, B. Bojovic, V. Vukcevic, P. Belicev, S. Pavlovic, Z. Vasiljevic-Pokrajcic, and M. Ostojic, "A novel mobile transtelephonic system with synthesized 12-lead ECG," IEEE Trans. Inform. Technol. Biomed., vol. 8, pp. 428-438, Dec. 2004.

[13] L. Edenbrandt and O. Pahlm, "Vectorcardiogram synthesized from a 12 lead ecg: Superiority of the inverse dower matrix," J. Electrocardiol., vol. 21, p. 361, 1988.

[14] A. Hyvärinen, J. Karhunen, and E. Oja, Independent Component Analysis. Wiley-Interscience, 2001.

[15] R. Abeysekera, "Some physiologically meaningful features obtained from Fourier descriptors of vectorcardiograph," "IEEE Eng. Med. Biol. Mag.", vol. 10, pp. 58-63, 1991.

[16] The MIT-BIH Noise Stress Test Database. [Online]. Available: http://www.physionet.org/physiobank/database/nstdb/

[17] B. D. Moor, Database for the Identification of Systems (DaISy). [Online]. Available: http://homes.esat.kuleuven.be/ smc/daisy/

[18] R. Sameni, M. B. Shamsollahi, C. Jutten, and M. Babaie-Zadeh, "Filtering Noisy ECG Signals Using the Extended Kalman Filter Based on a Modified Dynamic ECG Model," in Proceedings of the 32nd Annual International Conference on Computers in Cardiology, Lyon, France, September 25-28 2005, pp. 1017-1020. 